\title{
SELF-ADJOINT RESTRICTIONS OF MAXIMAL OPERATOR ON GRAPH
}

\author{
L.K. ZHAPSARBAYEVA, B.E. KANGUZHIN, M.N. KONYRKULZHAYEVA
}

\begin{abstract}
In the work we study differential operators on arbitrary geometric graphs without loops. We extend the known results for differential operators on an interval to the differential operators on the graphs. In order to define properly the maximal operator on a given graph, we need to choose a set of boundary vertices. The non-boundary vertices are called interior vertices. We stress that the maximal operator on a graph is determined not only by the given differential expressions on the edges, but also by the Kirchhoff conditions at the interior vertices of the graph. For the introduced maximal operator we prove an analogue of the Lagrange formula. We provide an algorithm for constructing adjoint boundary forms for an arbitrary set of boundary conditions. In the conclusion of the paper, we present a complete description of all self-adjoint restrictions of the maximal operator.
\end{abstract}

Keywords: Directed graph, Kirchhoff conditions, self-adjoint restriction of an operator, maximal operator

Mathematics Subject Classification: 34B45, 34L20

\section{INTRODUCTION}

The work is devoted to linear differential operators on graphs. The spectral theory of differential operators on manifolds of net kind was studied in works by Yu.V. Pokornyi and its pupils [1]-[5]. Among the recent publications devoted to various aspects of the theory on the inverse problems on graphs, we mention works [6]-[10]. In works [11]-[14], there were studied spectral properties of differential operators on equilateral quantum star-like graphs. In this paper the Lagrange formula was obtained for a differential operator defined on an arbitrary geometric graph, in contrast to work [12, where a similar relation was given for a simple stargraph. There was given the complete description of all self-adjoint restrictions on the maximal operator defined on the graph. The mentioned results are new, earlier similar facts were known for differential operators on a segment [15].

\section{BASIC NOTIONS}

Assume that we are given a directed graph $\Im=\{\mathcal{V}, \mathcal{E}\}$, where $\mathcal{V}$ is the set of vertices and $\mathcal{E}$ is the set of edges. The number of the vertices of the graph is denoted by $N$, while the vertices are indexed by the numbers $1, \ldots, N$. The boundary vertices are denoted by $\Gamma$. The vertices in the set $\mathcal{I}=\mathcal{V} \backslash \Gamma$ are called interior [16], [17]. The edge $e=[i, j], e \in \mathcal{E}$, connecting vertices $i$ and $j$ is directed from $i$ to $j$. One often denotes the vertex $i$ by $\partial_{-} e$, while $\partial_{+} e$ stands for $j$ [18. Without loss of generality, the length of each edge is assumed to be one. The set of the vertices corresponding to incoming edges at a vertex $i$ is denoted by $\mathcal{V}_{i}^{+}$. The set of the vertices outcoming from a vertex $i$ is denoted by $\mathcal{V}_{i}^{-}$. Let $\chi=\sum_{i \in \Gamma}\left(\left|\mathcal{V}_{i}^{+}\right|+\left|\mathcal{V}_{i}^{-}\right|\right)$. The set of

L.K. Zhapsarbayeva, B.E. Kanguzhin, M.N. Konyrkulzhayeva, Self-adjoint Restriction of MAXIMAL OPERATOR ON GRAPH.

(C)Zhapsarbayeva L.K., Kanguzhin B.E., Konyrkulzhayeva M.N. 2017.

The work is supported by MES of the Republic of Kazakhstan (grant 0757/GF4 and grant 0085/PTsF-14).

Submitted July 24, 2017. 
edges satisfying $\partial_{+} e=i$ is denoted by $\mathcal{E}_{i}^{+}$, while the set of edges satisfying $\partial_{-} e=i$ is denoted by $\mathcal{E}_{i}^{-}$. We assume that the graph has no loops.

\section{Definition of the MAXimal OPERATOR ON THE GRAPH}

For further purposes it is useful to introduce the space

$$
L_{2}(\Im) \doteqdot \prod_{e \in \mathcal{E}} L_{2}(e)
$$

with the elements

$$
\vec{Y}(\vec{X}) \doteqdot\left[y_{e}\left(x_{e}\right), e \in \mathcal{E}\right]^{T}
$$

where $\vec{X}=\left(x_{e}, e \in \mathcal{E}\right)$ and $\prod_{e \in \mathcal{E}}$ is the Cartesian product of the spaces, and with a finite norm

$$
\|\vec{Y}\|_{L_{2}(\Im)}=\sqrt{\sum_{e \in \mathcal{E}} \int_{e}\left|y_{e}\left(x_{e}\right)\right|^{2} d x_{e}} .
$$

In the same standard way we introduce the space

$$
W_{2}^{2}(\Im) \doteqdot \prod_{e \in \mathcal{E}} W_{2}^{2}(e)
$$

The operator $\Lambda_{\max }$ defined by the linear differential expressions

$$
\Lambda_{\max } y_{e}\left(x_{e}\right)=-y_{e}^{\prime \prime}\left(x_{e}\right)+q_{e}\left(x_{e}\right) y_{e}\left(x_{e}\right), x_{e} \in e \in \mathcal{E} \quad \text { or } \quad 0<x_{e}<1
$$

on the subspace $W_{2}^{2}(\Im)$, where for some $\beta_{k}$, in each interior vertex $k$ the Kirchhoff conditions

$$
\begin{aligned}
& y_{e}(1)=\beta_{k} \quad \text { for all } e \in \mathcal{E}_{k}^{+}, \\
& y_{e}(0)=\beta_{k} \quad \text { for all } e \in \mathcal{E}_{k}^{-}, \\
& \sum_{e \in \mathcal{E}_{k}^{+}} y_{e}^{\prime}(1)=\sum_{e \in \mathcal{E}_{k}^{-}} y_{e}^{\prime}(0),
\end{aligned}
$$

hold [19], is called the maximal operator. Here $\left\{q_{e}\left(x_{e}\right), x_{e} \in e \in \mathcal{E}, 0<x_{e}<1\right\}$ is the set of real continuous functions usually called potentials. We can exclude the set of unknown constants $\left\{\beta_{k}\right\}$ from conditions (2). Then the total amount of Kirchhoff conditions in the interior points is equal to $2|\mathcal{E}|-\chi$. In the next section we provide the Lagrange formula for the maximal operator $\Lambda_{\max }$.

\section{LAGRANGE FORMUla FOR DIFFERENTIAL OPERATOR ON GRAPH}

The Lagrange formula plays an important role in study of differential operators on a segment. In this section we provide an analogue of Lagrange formula for differential operators on graphs.

Lemma 1. For all function $\vec{Y}(x)=\left\{y_{e}\left(x_{e}\right), e \in \mathcal{E}\right\}, \vec{V}(x)=\left\{v_{e}\left(x_{e}\right), e \in \mathcal{E}\right\}$ in $W_{2}^{2}(\Im)$, the identity

$$
\sum_{e \in \mathcal{E}} \int_{e} \Lambda_{\text {max }} y_{e}(x) v_{e}(x) d x=\left.\sum_{e \in \mathcal{E}}\left[-y_{e}^{\prime}(x) v_{e}(x)+y_{e}(x) v_{e}^{\prime}(x)\right]\right|_{x=0} ^{x=1}+\sum_{e \in \mathcal{E}} \int_{e} y_{e}(x) \Lambda_{\max } v_{e}(x) d x
$$

holds true.

Proof. We consider the integral in the left hand side of relation (4)

$$
\int_{e} \Lambda_{\max } y_{e}(x) v_{e}(x) d x=\int_{e}\left(-y_{e}^{\prime \prime}(x)+q_{e}(x) y_{e}(x)\right) v_{e}(x) d x .
$$

The double integration by parts allows us to write the formula

$$
\int_{e} \Lambda_{\max } y_{e}(x) v_{e}(x) d x=\left.\left[-y_{e}^{\prime}(x) v_{e}(x)+y_{e}(x) v_{e}^{\prime}(x)\right]\right|_{x=0} ^{x=1}+\int_{e} y_{e}(x)\left(-v_{e}^{\prime \prime}(x)+q_{e}(x) v_{e}(x)\right) d x .
$$


This implies (4). The proof is complete.

Lemma 2. For all functions $\vec{Y}(x)=\left\{y_{e}\left(x_{e}\right), e \in \mathcal{E}\right\}, \vec{V}(x)=\left\{v_{e}\left(x_{e}\right), e \in \mathcal{E}\right\}$ in the domain of the maximal operator $\Lambda_{\max }$ the identity

$$
\begin{aligned}
\sum_{e \in \mathcal{E}} \int_{e} \Lambda_{\max } y_{e}(x) v_{e}(x) d x= & \sum_{\partial_{+} \in \Gamma}\left[-y_{e}^{\prime}(1) v_{e}(1)+y_{e}(1) v_{e}^{\prime}(1)\right] \\
& -\sum_{\partial_{-} \in \Gamma}\left[-y_{e}^{\prime}(0) v_{e}(0)+y_{e}(0) v_{e}^{\prime}(0)\right]+\sum_{e \in \mathcal{E}} \int_{e} y_{e}(x) \Lambda_{\max } v_{e}(x) d x
\end{aligned}
$$

holds.

Proof. Let $a$ be an interior vertex of the graph $\Im$. The set $\mathcal{E}_{a}^{+}$consists of edges $e$ directed to the vertex $a$, that is, the condition $\partial_{+} e=a$ holds. The set $\mathcal{E}_{a}^{-}$consists of edges $e$ directed out from the vertex $a$, that is, the condition $\partial_{-} e=a$ holds. In the general sum $\sum_{e \in \mathcal{E}}\left[-y_{e}^{\prime}(x) v_{e}(x)+\right.$ $\left.y_{e}(x) v_{e}^{\prime}(x)\right]\left.\right|_{x=0} ^{x=1}$ we select only the terms corresponding the interior vertex $a$. For an edge $e$ with the condition $\partial_{+} e=a$, the contribution of the vertex $a$ into the total sum

$$
\left.\sum_{e \in \mathcal{E}}\left[-y_{e}^{\prime}(x) v_{e}(x)+y_{e}(x) v_{e}^{\prime}(x)\right]\right|_{x=0} ^{x=1}
$$

is of the form

$$
\left[-y_{e}^{\prime}(1) v_{e}(1)+y_{e}(1) v_{e}^{\prime}(1)\right]
$$

In the same way for all edges in $\mathcal{E}_{a}^{+}$the contribution of the vertex $a$ into the total sum

$$
\left.\sum_{e \in \mathcal{E}}\left[-y_{e}^{\prime}(x) v_{e}(x)+y_{e}(x) v_{e}^{\prime}(x)\right]\right|_{x=0} ^{x=1}
$$

is of the form

$$
\sum_{e \in \mathcal{E}_{a}^{+}}\left[-y_{e}^{\prime}(1) v_{e}(1)+y_{e}(1) v_{e}^{\prime}(1)\right]
$$

For one edge $e$ with the condition $\partial_{-} e=a$, the contribution of the vertex $a$ in the total sum

$$
\left.\sum_{e \in \mathcal{E}}\left[-y_{e}^{\prime}(x) v_{e}(x)+y_{e}(x) v_{e}^{\prime}(x)\right]\right|_{x=0} ^{x=1}
$$

is

$$
\left[y_{e}^{\prime}(0) v_{e}(0)-y_{e}(0) v_{e}^{\prime}(0)\right]
$$

In the same way, for all edges in $\mathcal{E}_{a}^{-}$the contribution of the vertex $a$ in the total sum

$$
\left.\sum_{e \in \mathcal{E}}\left[-y_{e}^{\prime}(x) v_{e}(x)+y_{e}(x) v_{e}^{\prime}(x)\right]\right|_{x=0} ^{x=1}
$$

is

$$
\sum_{e \in \mathcal{E}_{a}^{-}}\left[y_{e}^{\prime}(0) v_{e}(0)-y_{e}(0) v_{e}^{\prime}(0)\right]
$$

Thus, the total contribution of the vertex $a$ into the sum

$$
\left.\sum_{e \in \mathcal{E}}\left[-y_{e}^{\prime}(x) v_{e}(x)+y_{e}(x) v_{e}^{\prime}(x)\right]\right|_{x=0} ^{x=1}
$$

can be written as

$$
I_{a}=\sum_{e \in \mathcal{E}_{a}^{+}}\left[-y_{e}^{\prime}(1) v_{e}(1)+y_{e}(1) v_{e}^{\prime}(1)\right]+\sum_{e \in \mathcal{E}_{a}^{-}}\left[y_{e}^{\prime}(0) v_{e}(0)-y_{e}(0) v_{e}^{\prime}(0)\right] .
$$


Taking into consideration Kirchhoff conditions (2) for the functions $\vec{Y}(x)=\left\{y_{e}\left(x_{e}\right), e \in \mathcal{E}\right\}$, $\vec{V}(x)=\left\{v_{e}\left(x_{e}\right), e \in \mathcal{E}\right\}$, we rewrite the latter expression as

$$
I_{a}=\sum_{e \in \mathcal{E}_{a}^{+}}\left[-y_{e}^{\prime}(1) \tilde{\beta}+\beta v_{e}^{\prime}(1)\right]+\sum_{e \in \mathcal{E}_{a}^{-}}\left[y_{e}^{\prime}(0) \tilde{\beta}-\beta v_{e}^{\prime}(0)\right],
$$

where $v_{e}(1)=\tilde{\beta}$ as $e \in \mathcal{E}_{a}^{+}$, and also $v_{e}(0)=\tilde{\beta}$ as $e \in \mathcal{E}_{a}^{-}$. Taking into consideration Kirchhoff conditions $(3)$ for the functions $\vec{Y}(x)=\left\{y_{e}\left(x_{e}\right), e \in \mathcal{E}\right\}, \vec{V}(x)=\left\{v_{e}\left(x_{e}\right), e \in \mathcal{E}\right\}$, we rewrite the expression $\bar{I}_{a}$ as

$$
I_{a}=\tilde{\beta}\left[-\sum_{e \in \mathcal{E}_{a}^{+}} y_{e}^{\prime}(1)+\sum_{e \in \mathcal{E}_{a}^{-}} y_{e}^{\prime}(0)\right]+\beta\left[\sum_{e \in \mathcal{E}_{a}^{+}} v_{e}^{\prime}(1)-\sum_{e \in \mathcal{E}_{a}^{-}} v_{e}^{\prime}(0)\right]=0 .
$$

The proof is complete.

Lemma 2 implies that the total contributions of the interior vertices in integrated terms (5) are zero. In other words, integrated terms (5) involve only the contributions of the boundary vertices. According monograph [15], such formulae are called Lagrange formulae. Formula (5) can be generalized as follows.

For $k=1, \ldots, 2 \chi$ we consider the boundary forms

$$
U_{k}(\vec{Y})=\sum_{\partial_{+} e \in \Gamma}\left[\alpha_{e k} y_{e}(1)+\beta_{e k} y_{e}^{\prime}(1)\right]+\sum_{\partial_{-} \in \Gamma}\left[\alpha_{e k} y_{e}(0)+\beta_{e k} y_{e}^{\prime}(0)\right]
$$

where $\alpha_{e k}, \beta_{e k}$ are some constants.

Theorem 1 (Lagrange formula). Let $\left\{U_{1}, \ldots, U_{2 \chi}\right\}$ be a set of linear independent boundary forms $\left\{T_{1}, \ldots, T_{2 \chi}\right\}$ such that for all functions $\vec{Y}(x)=\left\{y_{e}\left(x_{e}\right), e \in \mathcal{E}\right\}, \vec{V}(x)=\left\{v_{e}\left(x_{e}\right), e \in \mathcal{E}\right\}$ in the domain of the maximal operator $\Lambda_{\max }$ the identity

$$
\begin{aligned}
\sum_{e \in \mathcal{E}} \int_{e} \Lambda_{\max } y_{e}(x) v_{e}(x) d x= & U_{1}(\vec{Y}) T_{2 \chi}(\vec{V})+U_{2}(\vec{Y}) T_{2 \chi-1}(\vec{V})+\ldots \\
& +U_{2 \chi}(\vec{Y}) T_{1}(\vec{V})+\sum_{e \in \mathcal{E}} \int_{e} y_{e}(x) \Lambda_{\max } v_{e}(x) d x
\end{aligned}
$$

holds.

Proof. We introduce the difference

$$
R(\vec{Y}, \vec{V})=\sum_{e \in \mathcal{E}} \int_{e} \Lambda_{\max } y_{e}(x) v_{e}(x) d x-\sum_{e \in \mathcal{E}} \int_{e} y_{e}(x) \Lambda_{\max } v_{e}(x) d x .
$$

According Lemma 2, this difference can be represented as

$$
R(\vec{Y}, \vec{V})=\sum_{\partial_{+} e \in \Gamma}\left[-y_{e}^{\prime}(1) v_{e}(1)+y_{e}(1) v_{e}^{\prime}(1)\right]-\sum_{\partial_{-} \in \Gamma}\left[-y_{e}^{\prime}(0) v_{e}(0)+y_{e}(0) v_{e}^{\prime}(0)\right] .
$$

Thus, this difference is expressed in terms of $2 \chi$ boundary values $\left\{y_{e}(1), y_{e}^{\prime}(1), \partial_{+} e \in \Gamma\right\}$, $\left\{y_{e}(0), y_{e}^{\prime}(0), \partial_{-} e \in \Gamma\right\}$. This set of boundary values is a $2 \chi$ linearly independent system. This is why the mentioned set of boundary values can be expressed as a linear combination of arbitrary boundary forms $\left\{U_{1}, \ldots, U_{2 \chi}\right\}$ satisfying the assumptions of Theorem 1 . To prove this, relations (6) are to be considered as a system of linear algebraic equations w.r.t. $\left\{y_{e}(1), y_{e}^{\prime}(1), \partial_{+} e \in \Gamma\right\}$, 
$\left\{y_{e}(0), y_{e}^{\prime}(0), \partial_{-} e \in \Gamma\right\}$. solving system (6) w.r.t. $\left\{y_{e}(1), y_{e}^{\prime}(1), \partial_{+} e \in \Gamma\right\},\left\{y_{e}(0), y_{e}^{\prime}(0), \partial_{-} e \in\right.$ $\Gamma\}$, we obtain

$$
\begin{aligned}
& y_{e}(1)=\sum_{k=1}^{2 \chi} \gamma_{e k} U_{k}(\vec{Y}), \partial_{+} e \in \Gamma, \\
& y_{e}^{\prime}(1)=\sum_{k=1}^{2 \chi} \epsilon_{e k} U_{k}(\vec{Y}), \partial_{+} e \in \Gamma, \\
& y_{e}(0)=\sum_{k=1}^{2 \chi} \gamma_{e k} U_{k}(\vec{Y}), \partial_{-} e \in \Gamma, \\
& y_{e}^{\prime}(0)=\sum_{k=1}^{2 \chi} \epsilon_{e k} U_{k}(\vec{Y}), \partial_{-} e \in \Gamma .
\end{aligned}
$$

We substitute these expressions into the difference:

$$
\begin{aligned}
R(\vec{Y}, \vec{V})= & \sum_{\partial_{+} e \in \Gamma}\left[-\left(\sum_{k=1}^{2 \chi} \epsilon_{e k} U_{k}(\vec{Y})\right) v_{e}(1)+\left(\sum_{k=1}^{2 \chi} \gamma_{e k} U_{k}(\vec{Y})\right) v_{e}^{\prime}(1)\right] \\
& -\sum_{\partial_{-} \in \in \Gamma}\left[-\left(\sum_{k=1}^{2 \chi} \epsilon_{e k} U_{k}(\vec{Y})\right) v_{e}(0)+\left(\sum_{k=1}^{2 \chi} \gamma_{e k} U_{k}(\vec{Y})\right) v_{e}^{\prime}(0)\right] .
\end{aligned}
$$

Now it remains to group the right hand side of the latter identity w.r.t. $\left\{U_{k}(\vec{Y}\}\right.$. We obtain:

$$
R(\vec{Y}, \vec{V})=\sum_{k=1}^{2 \chi} U_{k}(\vec{Y})\left\{\sum_{\partial_{+} \in \in \Gamma}\left[-\epsilon_{e k} v_{e}(1)+\gamma_{e k} v_{e}^{\prime}(1)\right]+\sum_{\partial_{-} \in \Gamma}\left[\epsilon_{e k} v_{e}(0)-\gamma_{e k} v_{e}^{\prime}(0)\right]\right\} .
$$

In the latter expressions we denote the sums in the square brackets by

$$
T_{2 \chi-k+1}(\vec{V})=\sum_{\partial_{+} \in \in}\left[-\epsilon_{e k} v_{e}(1)+\gamma_{e k} v_{e}^{\prime}(1)\right]+\sum_{\partial_{-} \in \Gamma}\left[\epsilon_{e k} v_{e}(0)-\gamma_{e k} v_{e}^{\prime}(0)\right]
$$

to obtain the desired formula

$$
\sum_{e \in \mathcal{E}} \int_{e} \Lambda_{\max } y_{e}(x) v_{e}(x) d x=\sum_{k=1}^{2 \chi} U_{k}(\vec{Y}) T_{2 \chi-k+1}(\vec{V})+\sum_{e \in \mathcal{E}} \int_{e} y_{e}(x) \Lambda_{\max } v_{e}(x) d x .
$$

Formula (7) is called Lagrange formula.

Theorem 1 implies immediately the following corollary.

Corollary 1. Let $\Lambda$ be a restriction of the operator $\Lambda_{\max }$ on the domain

$$
D(\Lambda)=\left\{\vec{Y} \in D\left(\Lambda_{\max }\right): U_{k}(\vec{Y})=0, \ldots, U_{\chi}(\vec{Y})=0\right\} .
$$

Then the adjoint operator $\Lambda^{*}$ is also the restriction of the operator $\Lambda_{\text {max }}$ on the domain

$$
D\left(\Lambda^{*}\right)=\left\{\vec{V} \in D\left(\Lambda_{\max }\right): T_{k}(\vec{V})=0, \ldots, T_{\chi}(\vec{V})=0\right\}
$$

and for all $\vec{Y} \in D(\Lambda)$ and $\vec{V} \in D\left(\Lambda^{*}\right)$ the identity

$$
\sum_{e \in \mathcal{E}} \int_{e} \Lambda y_{e}(x) v_{e}(x) d x=\sum_{e \in \mathcal{E}} \int_{e} y_{e}(x) \Lambda^{*} v_{e}(x) d x
$$

holds true. 


\section{Self-Adjoint Restrictions of maximal operator $\Lambda_{\max }$}

In this section we describe completely all self-adjoint restrictions of the operator $\Lambda_{\max }$. We first introduce the minimal restriction $\Lambda_{0}$ of the operator $\Lambda_{\max }$. By $D\left(\Lambda_{0}\right)$ we denote the set of all functions $\vec{Y}(x)$ in $D\left(\Lambda_{\max }\right)$ satisfying the conditions

$$
\begin{array}{llll}
y_{e}(1)=0, & y_{e}^{\prime}(1)=0 & \text { as } & \partial_{+} e \in \Gamma \\
y_{e}(0)=0, & y_{e}^{\prime}(0)=0 & \text { as } & \partial_{-} e \in \Gamma
\end{array}
$$

We introduce the minimal restriction $\Lambda_{0}$ by the formula

$$
\Lambda_{0} \vec{Y}=\Lambda_{\max } \vec{Y}, \quad \vec{Y} \in D\left(\Lambda_{0}\right)
$$

The following statements hold.

I) for all elements $\vec{Y} \in D\left(\Lambda_{0}\right), \vec{V} \in D\left(\Lambda_{\max }\right)$ the relation

$$
\left\langle\Lambda_{0} \vec{Y}, \vec{V}\right\rangle=\left\langle\vec{Y}, \Lambda_{\max } \vec{V}\right\rangle
$$

holds true;

II) for all elements $\vec{Y}, \vec{V} \in D\left(\Lambda_{0}\right)$ the identity

$$
\left\langle\Lambda_{0} \vec{Y}, \vec{V}\right\rangle=\left\langle\vec{Y}, \Lambda_{0} \vec{V}\right\rangle
$$

holds true.

Identity 10$)$ implies the operator inclusion $\Lambda_{\max } \subset \Lambda_{0}^{*}$.

In order to study the properties of the minimal operator, it is convenient to introduce the operators $\Lambda_{1}$ and $\Lambda_{2}$ being the restrictions of the maximal operator $\Lambda_{\max }$. Let

$$
D\left(\Lambda_{1}\right)=\left\{\vec{Y} \in D\left(\Lambda_{\max }\right): y_{e}(1)=0 \quad \text { as } \quad \partial_{+} e \in \Gamma, \quad y_{e}(0)=0 \quad \text { as } \quad \partial_{-} e \in \Gamma\right\}
$$

and $\Lambda_{1} \vec{Y}(x)=\Lambda_{\max } \vec{Y}(x)$ as $\vec{Y} \in D\left(\Lambda_{1}\right)$.

Let

$$
D\left(\Lambda_{2}\right)=\left\{\vec{Y} \in D\left(\Lambda_{\max }\right): y_{e}^{\prime}(1)=0 \quad \text { as } \quad \partial_{+} e \in \Gamma, \quad y_{e}^{\prime}(0)=0 \quad \text { as } \quad \partial_{-} e \in \Gamma\right\}
$$

and $\Lambda_{2} \vec{Y}(x)=\Lambda_{\max } \vec{Y}(x)$ as $\vec{Y} \in D\left(\Lambda_{2}\right)$.

Assumption 1. For each function $\vec{F}(x)$ in $L_{2}(\Im)$ the equation

$$
\Lambda_{i} \vec{Y}(x)=\vec{F}(x), \quad i=1,2
$$

has the unique solution in $D\left(\Lambda_{i}\right), i=1,2$.

Remark 1. Operator equation (11) on the set $D\left(\Lambda_{i}\right), i=1,2$, is equivalent to the system of linear second order differential equations on the set of edges $\mathcal{E}$ with $2|\mathcal{E}|-\chi$ Kirchhoff conditions in the interior vertices $\mathcal{I}$ and $\chi$ conditions at the boundary vertices $\Gamma$. Thus, there arises a system of inhomogeneous linear second order differential equations on the set of edges $\mathcal{E}$ and the general solution involves $2|\mathcal{E}|$ constants. These constants are determined by $(2|\mathcal{E}|-\chi)+\chi=2|\mathcal{E}|$ linear conditions. Therefore, we can write some determinant $D_{i}, i=1,2$, of the dimension $2|\mathcal{E}|$. Then the unique solvability of equations (11) is equivalent to the fact that the determinant $D_{i}$, $i=1,2$, is non-zero.

Following monograph [15], we formulate the next lemmata.

Lemma 3. Let $\Lambda_{\max }$ be the maximal operator on the graph $\Im$ introduced in Section 3 and let $\vec{F}(x)$ be the function in $L_{2}(\Im)$. If Assumption 1 holds, then equation

$$
\Lambda_{\max } \vec{Y}(x)=\vec{F}(x)
$$

has a solution $\vec{Y}(x)$ satisfying conditions (9) if and only if $\vec{F}(x)$ are orthogonal to all elements $\operatorname{Ker} \Lambda_{\max }$. 
Proof. According Assumption 1, by $\vec{Y}(x)$ we denote the unique solution to operator equation 11). By $\vec{V}_{1}, \ldots, \vec{V}_{\chi}$ we denote the fundamental system of solutions to the homogeneous operator equation $\Lambda_{\max } \vec{V}=0$ satisfying the condition: all boundary forms $v_{k l}(1)$ as $\partial_{+} e \in \Gamma$ and $v_{k l}(0)$ as $\partial_{-} e \in \Gamma$ except one vanish, while one form is equal to 1 . Such fundamental system exists. This is implied by Remark 1 since the solvability condition is equivalent to the non-vanishing of the determinant $D_{1}$.

Applying Lagrange formula to the functions $\vec{Y}(x)$ and $\vec{V}_{k}(x)$, we obtain

$$
\left\langle\vec{F}, \vec{V}_{k}\right\rangle=\left\langle\Lambda_{\max } \vec{Y}, \vec{V}_{k}\right\rangle=\left\langle\vec{Y}, \Lambda_{\max } \vec{V}_{k}\right\rangle .
$$

By $\Lambda_{\text {max }} \vec{V}_{k}=0$. Moreover, the belonging $\vec{Y} \in D\left(\Lambda_{1}\right)$ implies

$$
\sum_{\partial_{+} e \in \Gamma} y_{e}(1) v_{k l}^{\prime}(1)-\sum_{\partial_{-} \in \Gamma} y_{e}(0) v_{k l}^{\prime}(0)=0
$$

Therefore, the formula 12 becomes

$$
\left\langle\vec{F}, \vec{V}_{k}\right\rangle=-\sum_{\partial_{+} \in \Gamma} y_{e}^{\prime}(1) v_{k l}(1)+\sum_{\partial_{-} \in \Gamma} y_{e}^{\prime}(0) v_{k l}(0)=\left\{\begin{array}{rll}
-y_{e}^{\prime}(1) & \text { if } \quad v_{k l}(1)=1, \\
y_{e}^{\prime}(0) & \text { if } \quad v_{k l}(0)=1 .
\end{array}\right.
$$

Relations 13 the statement of the lemma: identities 90 hold true if and only if $\left\langle\vec{F}, \vec{V}_{k}\right\rangle=0$, $k=1, \ldots, \chi$. Thus, $\vec{F}(x)$ is orthogonal to all solutions of the equation $\Lambda_{\max } \vec{V}=0$.

Lemma 4. Under Assumption 1, for all numbers $\alpha_{e}, \beta_{e}$ as $\partial_{+} e \in \Gamma$ and $\alpha_{e}$, $\beta_{e}$ as $\partial_{-} e \in \Gamma$ there exists a function $\vec{Y}(x) \in D\left(\Lambda_{\text {max }}\right)$ satisfying the conditions

$$
\begin{aligned}
& y_{e}^{\prime}(1)=\beta_{e}, y_{e}(1)=\alpha_{e} \quad \text { as } \quad \partial_{+} e \in \Gamma, \\
& y_{e}^{\prime}(0)=\beta_{e}, y_{e}(0)=\alpha_{e} \quad \text { as } \quad \partial_{-} e \in \Gamma .
\end{aligned}
$$

Proof. First we prove Lemma 4 for the case when $\alpha_{e}=0$. We choose $\vec{F}(x)$ in $L_{2}(\Im)$ so that

$$
\left\langle\vec{F}, \vec{V}_{k}\right\rangle=\left\{\begin{array}{rll}
-\beta_{e} & \text { if } & v_{k l}(1)=1 \\
\beta_{e} & \text { if } & v_{k l}(0)=1
\end{array}\right.
$$

where $\vec{V}_{k}, k=1, \ldots, \chi$ is the fundamental system of solutions used in the proof of Lemma 3 . Such element exists and it belongs to $\operatorname{Ker} \Lambda_{\max }$. Indeed, if we let

$$
\vec{F}=\sum_{k=1}^{\chi} \mu_{k} \vec{V}_{k}
$$

conditions (14) become a system of equations w.r.t. the constants $\mu_{1}, \ldots, \mu_{\chi}$. The determinant of this system is the Gram determinant of linearly independent functions $\vec{V}_{1}, \ldots, \vec{V}_{\chi}$. Therefore, it is non-zero. By $\vec{V}$ we denote the solution to equation $\Lambda_{1} \vec{V}=\vec{F}$. Then the Lagrange formula implies

$$
\begin{array}{lll}
\vec{V}^{\prime}(1)=\beta_{e} & \text { as } & \partial_{+} e \in \Gamma, \\
\vec{V}^{\prime}(0)=\beta_{e} & \text { as } & \partial_{-} e \in \Gamma .
\end{array}
$$

Thus, the constructed function $\vec{V}(x) \in D\left(\Lambda_{\max }\right)$ is such that

$$
\begin{array}{lll}
\vec{V}^{\prime}(1)=\beta_{e}, \vec{V}(1)=0 & \text { as } & \partial_{+} e \in \Gamma, \\
\vec{V}^{\prime}(0)=\beta_{e}, \vec{V}(0)=0 & \text { as } & \partial_{-} e \in \Gamma .
\end{array}
$$

Swapping the sets $\alpha_{e}$ and $\beta_{e}$ as well as the operators $\Lambda_{1}$ and $\Lambda_{2}$, we complete the proof.

Now we are in position to formulate a statement on the minimal operator $\Lambda_{0}$. 
Lemma 5. $\Lambda_{0} \subset \Lambda_{0}^{*}=\Lambda_{\max }, \Lambda_{\max }^{*}=\Lambda_{0}$.

Lemma 5 can be proved exactly in the same way as Statement V.17 in monograph [15].

The main theorem of this section is as follows.

Theorem 2. Under Assumption 1, each self-adjoint extension $\Lambda$ of the operator $\Lambda_{0}$ is determined by linearly independent $k=1, \ldots, \chi$ boundary conditions of form

$$
U_{k}(\vec{Y})=\sum_{\partial_{+} e \in \Gamma}\left[\alpha_{e k} y_{e}(1)+\beta_{e k} y_{e}^{\prime}(1)\right]+\sum_{\partial_{-} \in \Gamma}\left[\alpha_{e k} y_{e}(0)+\beta_{e k} y_{e}^{\prime}(0)\right]=0
$$

where $\alpha_{e k}, \beta_{e k}$ are some constants, and

$$
\sum_{\partial_{+} e \in \Gamma}\left[\alpha_{e j} \bar{\beta}_{e k}-\bar{\alpha}_{e k} \beta_{e j}\right]=\sum_{\partial_{-} \in \Gamma}\left[\alpha_{e j} \bar{\beta}_{e k}-\bar{\alpha}_{e k} \beta_{e j}\right]
$$

as $j, k=1, \ldots, \chi$.

And vice versa, each linearly independent boundary conditions (15) satisfying relations (16) define a domain for some self-adjoint extension $\Lambda$ of the operator $\Lambda_{0}$ if Assumption 1 holds true.

Proof. Following the lines in the proof of Theorem 5 in [15, Sect. 18], we introduce the functions $\vec{V}_{1}, \ldots, \vec{V}_{\chi}$. More precisely, $\vec{V}_{k}$ are in the domain of $D\left(\Lambda_{\max }\right)$ with the conditions

$$
v_{k e}^{\prime}(0)=-\alpha_{e k}, v_{k e}(0)=\beta_{e k} \quad \text { as } \quad \partial_{-} e \in \Gamma .
$$

According Lemma 4, such solutions exist. Then conditions (15) for $j, k=1, \ldots, \chi$ become

$$
U_{k}(\vec{Y})=\sum_{\partial_{+} e \in \Gamma}\left[y_{e}(1) v_{k e}^{\prime}(1)-y_{e}^{\prime}(1) v_{k e}(1)\right]-\sum_{\partial_{-} \in \Gamma}\left[y_{e}(0) v_{k e}^{\prime}(0)-y_{e}^{\prime}(0) v_{k e}(0)\right]=0 .
$$

According the results of monograph [15], boundary conditions (15) define the domain of a selfadjoint extension $\Lambda$ of the operator $\Lambda_{0}$ if and only if all functions $\vec{V}_{1}, \ldots, \vec{V}_{\chi}$ defined by (17) also satisfy the relations

$$
U_{k}\left(\vec{Y}_{j}\right)=0, \quad k, j=1, \ldots, \chi
$$

The proof is complete.

\section{BIBLIOGRAPHY}

1. Yu.V. Pokornyi. On spectra of some problems on graphs // Uspekhi Matem. Nauk. 42:4, 128-129 (1987). (in Russian).

2. O.M. Penkin, Yu.V. Pokornyi. On a boundary value problem on a graph // Differ. Uravn. 24:4, 701-703 (1988). (in Russian).

3. Yu.V. Pokornyi, V.L. Pryadiev, A. Al-Obeid. Oscillation properties of the spectrum of a boundary value problem on a graph // Matem. Zametki. 60:3, 468-469 (1996). [Math. Notes. 60:3, 351-353 (1996).]

4. Yu.V. Pokornyi, V.L. Pryadiev. Some problems of the qualitative Sturm-Liouville theory on a spatial network // Uspekhi Matem. Nauk. 59:6, 115-150 (2004). [Russ. Math. Surv. 59:3, 515-552 (2004).]

5. Yu.V. Pokornyi, O.M. Penkin, V.L. Pryadiev, A.V. Borovskikh, K.P. Lazarev, S.A. Shabrov. Differential equations on geometric graphs. Fizmatlit, Moscow (2005). (in Russian).

6. M.I. Belishev. Boundary spectral inverse problem on a class of graphs (trees) by the BC methods // Inverse Probl. 35:10, 4069-4088 (2004).

7. R. Carlson. Inverse eigenvalue problems on directed graphs // Trans. Amer. Math. Soc. 351:10, 101-121 (1999).

8. P. Kurasov, F. Stenberg. On the inverse scattering problem on branching graphs // J. Phys. A. Math. Gen. 35:1, 101-122 (2002). 
9. M. Ramirez Jorge. Green's functions for Sturm-Liouville problems on directed tree graphs // Revista Colomb. Matemáticas. 46:1, 15-25 (2012).

10. V.A. Yurko. On recovering Sturm-Liouville operators on graphs // Matem. Zamet. 79:4, 619-630 (2006). [Math. Notes. 79:4, 572-582 (2006).]

11. M. Astudillo, P. Kurasov, M. Usman. $\mathcal{R} \mathcal{T}$-symmetric Laplace operators on star graphs: real spectrum and selfadjointness // Adv. Math. Phys. 2015, id 649795 (2015).

12. P. Kurasov, M. Garjiani. Quantum graphs: PT-symmetry and reflection symmetry of the spectrum // J. Math. Phys. 58, id 023506 (2017).

13. M. Znojil. Quantum star-graph analogues of PT-symmetric square wells // Can. J. Phys. 90:12, 1287-1293 (2012).

14. M. Znojil. Quantum star-graph analogues of PT-symmetric square wells: Part II. Spectra // Can. J. Phys. 93:7, 765-768 (2012).

15. M.A. Naimark. Linear differential operators. Nauka, Moscow (1969). [Part I: Elementary theory of linear differential operators, Frederick Ungar Publ. Co., New York (1967); Part II: Linear differential operators in Hilbert space, Frederick Ungar Publ. Co., New York (1968)].

16. S. Tsoi, S.M. Tskhay. Applied graph theory. Nauka, Alma-Ata (1971). (in Russian).

17. F. Harary. Graph theory. Addison-Wesley Publ. Co., Reading (1969).

18. O. Post. Spectral analysis on graph-like spaces. Lect. Notes Math. 2039. Springer, Berlin (2012).

19. N.A. Afanasieva, L.P. Bulot. Electrotechnique and electronics. Inst. Cold Biotechnology, ITMO Univ. (2010) (in Russian).

Lyailya Kurmantayevna Zhapsarbayeva,

Al-Farabi Kazakh National University,

Al-Farabi av. 71,

050040, Almaty, Kazakhstan

E-mail: leylazhk67@gmail.com

Baltabek Esmatovich Kanguzhin,

Al-Farabi Kazakh National University,

Al-Farabi av. 71,

050040, Almaty, Kazakhstan

E-mail: kanbalta@mail.ru

Maral Nurlanovna Konyrkulzhayeva, Al-Farabi Kazakh National University, Al-Farabi av. 71, 050040, Almaty, Kazakhstan

E-mail: maralkulzha@gmail.com 